\title{
Padrão de actividade física. Estudo em crianças de ambos os sexos do $4 .^{\circ}$ ano de escolaridade
}

\author{
Luísa Magalhães \\ José Maia \\ Rui Silva \\ André Seabra
}

\author{
Faculdade de Ciências do Desporto e de Educação Física \\ Universidade do Porto \\ Portugal
}

\section{RESUMO}

O presente estudo visa conhecer os níveis de actividade física no tempo de lazer e o padrão (ao nível do modo, da intensidade, da duração e da frequência) da actividade física habitual de crianças em contexto escolar. Foi seleccionada uma amostra de 120 crianças, de ambos os sexos, de 10 anos de idade (em média), a frequentar o $4^{\circ}$ ano de escolaridade em duas escolas do $1^{\circ}$ Ciclo do Ensino Básico de Vila Nova de Gaia. A avaliação da actividade física no tempo de lazer foi efectuada através do questionário de Godin e Shephard (21). Para avaliar a actividade física no tempo escolar, solicitou-se a 49 crianças o uso de um acelerómetro portátil (Tritrac-R3D) durante cinco dias. Os procedimentos estatísticos usados foram as medidas descritivas média, desvio-padrão, frequências absolutas e relativas; o tteste de medidas independentes e o teste não-paramétrico de Mann-Whitney. Os principais resultados foram os seguintes: (1) os rapazes apresentam um índice superior de actividade física no tempo de lazer, quando comparados com as raparigas, principalmente no que se refere à actividade física de intensidade elevada; (2) as crianças evidenciam um padrão de actividade física que se caracteriza pela aleatoriedade com oscilações na sua intensidade e duração; (3) as crianças evidenciam durante o período escolar um predomínio de actividade física de intensidade baixa, não realizando, no mínimo, 30 minutos de actividade física moderada a vigorosa diária; (4) os rapazes apresentam valores significativamente $(\mathrm{p}<0.05)$ mais elevados do que as raparigas de actividade física moderada a vigorosa no recreio; (5) as crianças, mesmo em sessões organizadas de Educação Física, apresentam um padrão de actividade física caracterizado pela sua baixa intensidade, não despendendo pelo menos $50 \%$ do tempo total da aula em actividade física moderada a vigorosa; (6) o dispêndio energético não expressa um comportamento diferente nas sessões organizadas de Educação Física e nos momentos de actividade livre de recreio.

Palavras-chave: Actividade física; crianças; $1 .^{\circ}$ ciclo do ensino básico, acelerometria.

\author{
ABSTRACT \\ Physical activity patterns. \\ A study in primary school children of both gender.
}

The present study is aimed to perceive the different standards of physical activity in leisure time and the pattern of physical activity (regarding its manner, intensity, duration and frequency) of children within school context. A group of 120 children of both sexes, around the age of ten, attending Year 4 was selected amongst two Primary Schools in Vila Nova de Gaia. To assess the physical activity within their free time it was used Godin and Shephard's (21) questionnaire. Forty-nine children were asked to wear a portable accelerometer (Tritrac-R3D) for five days in order to assess their physical activity at school. The statistical procedures used were an average descriptive gauge, a standard deviation, unconditional and comparative occurrence; the independent measurement $t$-test and the Mann-Whitney non-parametric test. The results and conclusions obtained through this study suggest that: (1) boys show a greater rate of activity during free time in comparison to girls, specially in what concerns to high vigour physical activity; (2) children clearly manifest a pattern of physical activity which is fortuitous and it varies in its intensity and duration; (3) during school time children have physical activities of low intensity with less than 30 minutes per day of it being moderate/vigorous; (4) in the playground boys are significantly more physically active than girls $(p<0.05)$; (5) even in planned PE sessions the standard of physical activity is of low intensity thus demonstrating children spend less than $50 \%$ of lessons performing moderate/vigorous exercises; (6) a change of behaviour is neither reflected in planned PE sessions nor in playground activity regardless of the energy outlay.

Keywords: physical activity, children, primary school, accelerometry. 


\section{INTRODUÇÃO}

Numa altura em que a qualidade de vida e a saúde se afiguram como dimensões prioritárias, pelo menos nas sociedades ditas desenvolvidas, a epidemiologia tem visto ampliado o seu campo de acção reflectido, por exemplo, no número crescente de estudos realizados a esse nível no âmbito da actividade física (AF). Surge como evidência científica que a AF é uma componente importante na adopção de um estilo de vida saudável, assim como preventivo de múltiplas doenças e morte prematura, com benefícios presentes na adolescência e durante o estado adulto $(10,16,36,38)$.

Em relação às idades pediátricas, apesar de persistir alguma incerteza científica, muitos autores acreditam: nos efeitos positivos da AF para a sua saúde e nas consequências adversas de uma vida sedentária (ver, por exemplo, 42); que muitos dos factores de risco de doenças crónicas se iniciam na infância $(13,61)$ e que comportamentos activos durante a infância podem influenciar o estilo de vida no adulto $(50,56)$. Há, inclusivamente, um documento de uma reconhecida instituição de saúde americana que conclui que uma grande percentagem da sua população infantil e adolescente é sedentária, o que contribui para uma "epidemia sem precedentes" de obesidade infantil que terá duplicado desde 1980 (63). Por outro lado, um estudo realizado por Freedman, Dietz, Srinivasan e Berenson (18) permitiu concluir que, de uma amostra de crianças com peso excessivo, entre os cinco e os quinze anos de idade, $61 \%$ apresentava, pelo menos, um factor de risco e $27 \%$, pelo menos, dois factores de risco de doenças cardiovasculares.

Há, de facto, indícios de que as crianças apresentam, actualmente, níveis inferiores de $\operatorname{AF}(9,22,56)$, como consequência da generalização dos meios de transporte e da forte atracção que revelam por actividades mais sedentárias na ocupação dos tempos livres (nomeadamente a ver televisão e a jogar no computador).

A busca de evidência científica para estes pressupostos tem motivado uma crescente preocupação em estudar os padrões de AF apresentados pelas crianças que, por sua vez, tem levantado questões metodológicas específicas quanto à sua avaliação, provavelmente, estando na origem de alguma ambiguidade nos resultados obtidos $(39,53)$. No entanto, é possível verificar-se um certo consenso sobre o facto de um estilo de vida activo e uma boa condição física, assim como o desenvolvimento de atitudes posi- tivas face à AF, se dever iniciar na infância, apresentando fortes probabilidades de se repercutir no futuro. Surgem, assim, diversas recomendações e programas que têm em vista a intervenção no aumento dos níveis de $\mathrm{AF}$ das crianças, com um enfoque particular na escola e na Educação Física (EF).

Em Portugal, poucos estudos foram realizados neste âmbito, desconhecendo-se, de forma detalhada, os padrões de AF das crianças e se estes são idênticos ou similares aos definidos em estudos com outras populações. É fundamental desenvolver trabalhos nesta área, muito especialmente ao nível do $1^{\circ} \mathrm{Ciclo}$ do Ensino Básico em que, considerando a vasta proporção de crianças envolvidas, é essencial avaliar o que a escola, em geral, tem projectado e realizado neste âmbito em relação a estas crianças e, em particular, a EF, enquanto área curricular obrigatória presente neste ciclo de ensino.

Assim, é objectivo central deste estudo conhecer os níveis de AF no tempo de lazer e as exigências (ao nível do modo, da intensidade, da duração e da frequência) da AF habitual, em contexto escolar, de uma amostra de crianças de ambos os sexos a frequentar o $4^{\circ}$ ano de escolaridade.

\section{MATERIAL E MÉTODOS}

\subsection{Amostra}

A amostra foi constituída por 58 raparigas e 62 rapazes com 10,0 $\pm 0,6$ anos de idade a frequentar o $4^{\circ}$ ano de escolaridade, em duas escolas do Concelho de Vila Nova de Gaia. Em ambas as escolas, todos os alunos da amostra frequentavam o horário duplo da manhã (das 8 às 13 horas, com 30 minutos de intervalo) e participavam numa aula semanal de Expressão e Educação Físico-Motora, leccionada por um professor licenciado em EF.

\subsection{Avaliação da actividade física nos tempos de lazer}

A avaliação da AF realizada nos tempos de lazer foi efectuada através do Questionário de Godin e Shephard (21), validado com adultos $(21,31)$ e determinada a sua fiabilidade com crianças e adolescentes a partir dos dez anos de idade (49). Este questionário foi igualmente administrado numa amostra da população portuguesa a 223 crianças de dez e onze anos de idade por Barbosa, Magalhães e Lopes (6).

O questionário de Godin e Shephard (21) pretende quantificar a AF realizada na ocupação dos tempos livres, durante sete dias. Os sujeitos registam o número de vezes, por semana, em que despendem 
mais de quinze minutos em actividades que são classificadas como leves (3 METs), moderadas (5 METs) ou extenuantes (9 METs). O score final obtém-se pela multiplicação da frequência em cada categoria pelo valor de MET respectivo e pela soma posterior dos produtos obtidos.

É de referir que a fiabilidade das respostas ao questionário foi avaliada através da administração repetida do questionário, após duas semanas, em 23 dos 120 sujeitos da amostra.

\subsection{Avaliação da actividade física no tempo escolar}

A avaliação da AF realizada durante o período escolar foi concretizada num subgrupo de 49 sujeitos, através de um acelerómetro portátil (Tritrac - R3D), durante os cinco dias da semana escolar.

Os resultados obtidos são possíveis de visualizar sob a forma de quadros ou de registos numéricos que apresentam dados individuais por minuto. Sendo um acelerómetro triaxial, os quadros permitem-nos a visualização de gráficos de movimentos em três eixos, consoante a sua orientação - X (antero-posterior), Y (vertical) e $Z$ (médio-lateral) - mas também referente ao vector resultante calculado através da seguinte fórmula: $\sqrt{ }\left(\mathrm{X}^{2}+\mathrm{Y}^{2}+\mathrm{Z}^{2}\right)$. A partir deste vector resultante, pode ainda representar-se um outro relativo às kilocalorias (Kcal) despendidas, através de uma fórmula de conversão existente no próprio aparelho (não referenciada no manual). A partir das Kcal despendidas, os resultados podem ser ainda convertidos em METs, através da seguinte fórmula: (Kcal em actividade*70) / peso em Kg do sujeito, por minuto.

\subsection{Procedimentos estatísticos}

A descrição das variáveis em estudo foi efectuada a partir das medidas descritivas média, desvio-padrão, frequências absolutas e relativas. A análise das diferenças foi efectuada a partir do t-teste de medidas independentes e, também, do teste não-paramétrico de Mann-Whitney.

Os cálculos foram realizados no programa Excel para o Windows 98 e no programa de estatística SPSS (Statistical Package for the Social Sciences), versão 10.0. O nível de significância foi mantido em $5 \%$.

\section{APRESENTAÇÃO E DISCUSSÃO DOS RESULTADOS}

\subsection{Níveis de actividade física no tempo de lazer}

Dadas as dificuldades inerentes à utilização do questionário na infância, é de referir que a consistência ou fiabilidade das suas respostas foi avaliada através da administração directa e repetida do questionário, após duas semanas, em 23 dos 120 sujeitos da amostra. Os coeficientes de correlação intra-classe obtidos situam-se entre 0.73 a 0.97 , tradutores de valores baixos de variância-erro, sobretudo quando considerados no substracto da aplicação de questionários de $\mathrm{AF}$, apontando, assim, para uma utilização promissora deste questionário em crianças deste nível etário.

Como se pode verificar no quadro $n .^{\circ} 1$, as raparigas, no seu conjunto, apresentam valores médios superiores, embora não sejam estatisticamente significativos, de AF leve e moderada, enquanto que os rapazes registam valores significativamente superiores de AF extenuante e, consequentemente, um score total, igualmente, mais elevado.

Quadro 1: AF leve, moderada, extenuante e valor compósito da AF de lazer, por sexo (média e desvio-padrão), valor da estatística te valor de $p$, resultantes da administração do questionário de Godin e Shephard.

\begin{tabular}{lcccc}
\multicolumn{7}{c}{ Raparigas ( $\mathrm{n}=58$ ) } & Rapazes $(\mathrm{n}=62)$ & & \\
Variáveis & Média $\pm \mathrm{DP}$ & Média $\pm \mathrm{DP}$ & $\mathrm{t}{ }^{1}$ & $\mathrm{p}$ \\
\hline AF Leve & $3.72 \pm 3.57$ & $2.77 \pm 3.25$ & 1.53 & 0.13 \\
AF Moderada & $4.24 \pm 2.10$ & $3.76 \pm 2.43$ & 1.16 & 0.25 \\
AF Extenuante & $1.52 \pm 1.78$ & $4.85 \pm 3.46$ & -6.57 & $\mathbf{0 . 0 0}$ \\
Score Total & $46.03 \pm 20.81$ & $70.81 \pm 27.06$ & -5.59 & $\mathbf{0 . 0 0}$
\end{tabular}

Os valores obtidos sugerem que estas crianças se envolvem em poucos períodos de AF semanal, com uma duração mínima de quinze minutos, pelo menos de forma contínua. No entanto, se atendermos à variabilidade encontrada nos valores registados, verifica-se que a magnitude do desvio-padrão é semelhante à da média. Isto ocorre pelo facto de haver respostas nulas nos diversos itens, ou seja, elementos da amostra, tanto do sexo feminino como masculino, que referiram não ter realizado qualquer período de AF dentro de cada uma das três categorias. Este é um facto que deve preocupar todos aqueles que podem desempenhar um papel na promoção de estilos mais activos das crianças que realizam pouca AF (família, professores, médicos, entre outros).

\footnotetext{
${ }^{1}$ Aspectos condicionadores da normalidade das distribuições implicaram que as mesmas variáveis fossem analisadas com o teste não-paramétrico de Mann-Whitney que confirmou os resultados anteriores.
} 
Os resultados obtidos no presente estudo estão de acordo com a ideia expressa por diversos investigadores (e.g. 43, 52) de que é possível encontrar diferenças nos níveis de AF quanto ao sexo. Sallis (46) refere que, a partir da análise de estudos baseados em inquérito, os rapazes são cerca de $14 \%$ mais activos do que as raparigas e que este padrão é similar quando avaliado através de métodos objectivos. Resultados concordantes foram igualmente encontrados por Bradley, McMurray, Harrell e Deng (11), Hovell, Sallis, Kolody e McKenzie (23), Myers, Strikmiller, Webber e Berenson (35), Riddoch, Mahoney, Murphy, Boreham e Cran (41) e Sallis, McKenzie, Elder, Hoy, Galati, Berry, Zive e Nader (52) quando referem que, enquanto a rapariga regista mais AF de intensidade baixa, o rapaz se envolve mais em AF de intensidade elevada.

Desconhece-se ainda se o mecanismo primário que fundamenta as diferenças encontradas nos níveis de AF, em função do sexo, é biológico ou decorre de distintos processos de socialização (44). É, de facto, possível encontrar argumentos plausíveis que se relacionam com crenças socioculturais e expectativas distintas para ambos os sexos (60). Há indícios, por exemplo, de que a família, primeiro agente de socialização, encoraja desde cedo preferencialmente os rapazes a realizar AF, transportando-os com mais frequência para os locais onde podem realizar actividade física ou desportiva (48). Não parece, no entanto, que a explicação se centre exclusivamente na influência complexa do envolvimento, devendo este ser considerado em interacção e correlação com o genótipo que pode, até, operar com genes diferentes nos dois sexos (26).

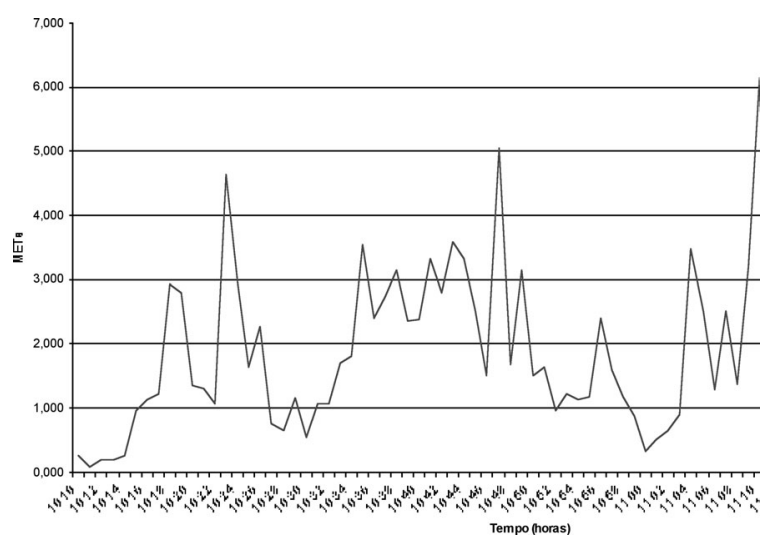

Figura 1: Representação gráfica dos valores registados em METs pelo Tritrac de um elemento do sexo feminino da amostra.

\subsection{Actividade física no tempo escolar}

A categorização da intensidade das actividades decorreu da necessidade sentida de utilizar um critério universalmente aceite e que permita a futura comparação de resultados obtidos em diferentes estudos, mesmo utilizando metodologias distintas. De facto, o MET (múltiplo da taxa metabólica de base correspondente a $3.5 \mathrm{mlO}_{2} \cdot \mathrm{Kg} \cdot{ }^{-1} \mathrm{~min}^{-1}$ ) é uma unidade universalmente aceite para expressar o dispêndio energético em função do peso corporal do sujeito (33). É ainda usual e consensual, a partir desta unidade, classificar as actividades como de baixa intensidade ( $\leq 3 \mathrm{METs}$ ), moderada (entre 3 e 7 METs) e vigorosa ( $\geq 7$ METs) (17).

Por outro lado, dada a constatação de que a criança não opta voluntariamente pela realização de AF vigorosa contínua, como, aliás, se confirma pela análise dos dados obtidos pelo acelerómetro na presente pesquisa (ver figuras 1 e 2), optámos pelo critério da duração total de AF de diferentes intensidades, acumulado, independentemente de ser realizada de forma contínua ou intermitente ao longo dos períodos de tempo em análise. $\mathrm{O}$ dispêndio energético e o valor acumulado da AF realizada, mesmo que de forma intermitente é, de facto, o critério considerado actualmente como o mais adequado para a avaliação da $\mathrm{AF}$ de crianças $(8,14,37)$, em detrimento da análise de períodos contínuos de AF com um registo de valores consistentemente acima de um determinado valor critério.

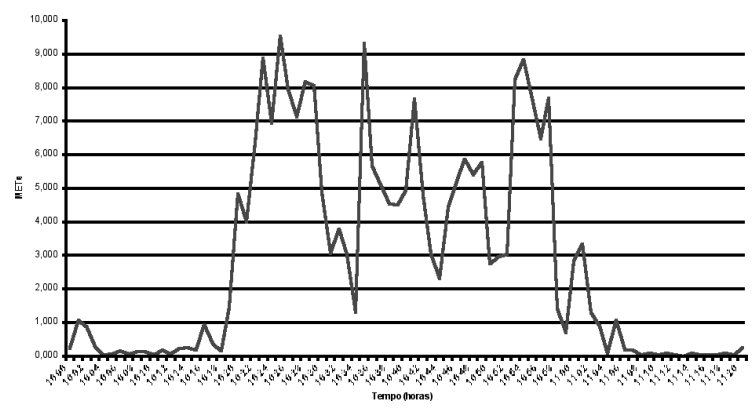

Figura 2: Representação gráfica dos valores registados em METs pelo Tritrac de um elemento do sexo masculino da amostra. 


\subsubsection{Características do padrão de actividade física das crianças da amostra}

Decorrente da análise das figuras 1 e 2, parece-nos ser importante destacar a aleatoriedade presente no padrão de AF evidenciado pelas crianças da amostra deste estudo. Esta complexidade e variabilidade é facilmente visível na representação gráfica dos valores obtidos durante o recreio, quando lhes é possível apresentar um comportamento mais espontâneo, em que se verifica, de forma mais ou menos notória, a natureza transitória da sua $\mathrm{AF}$, oscilando na sua intensidade com intervalos de duração irregular. Uma primeira análise exploratória dos dados obtidos permitiu-nos, desde logo, constatar que o exercício prolongado com níveis de intensidade elevados não é característico do padrão de AF habitual destas crianças, aliás, como já terá sido constatado por Saris (54) e por diversas investigações realizadas neste âmbito (e.g. 1, 4, 5). De facto, muitos dos estudos realizados para avaliar a AF habitual de crianças que concluem que estas são pouco activas, fazem-no na medida em que verificam que, geralmente, participam em poucos períodos de dez ou vinte minutos de AFMV, sempre acima de um determinado valor critério, de forma contínua $(7,12,20,45)$; o que, aliás, parece ser natural, dadas as características do seu padrão espontâneo de actividade.

\subsubsection{Resultados obtidos sobre o dia escolar}

No quadro 2 podemos verificar, em média, o tempo (em minutos) e correspondente proporção (em percentagem) despendida em actividades categorizadas pela intensidade, de acordo com os dados recolhidos pelo acelerómetro e onde se verifica que, na maior percentagem do tempo $(92.6 \pm 3.4 \%)$ passado na escola, as crianças se envolveram em actividades de baixa intensidade.
Quadro 2: Tempo (minutos] e proporção [\%] de AF de intensidade baixa, moderada e vigorosa, na totalidade da amostra (média e desvio-padrão) no dia escolar.

\begin{tabular}{lcccc} 
& \multicolumn{2}{c}{ Tempo [minutos] } & \multicolumn{2}{c}{ Proporção [\%] } \\
Variáveis & Média & Desvio-padrão & Média & Desvio-padrão \\
\hline AF Leve & 278.6 & 10.2 & 92.6 & 3.4 \\
AF Moderada & 20.0 & 8.7 & 6.6 & 2.9 \\
AF Vigorosa & 2.4 & 2.3 & 0.8 & 0.8 \\
AFMV & 22.4 & 10.2 & 7.4 & 3.4
\end{tabular}

Estes resultados parecem, assim, ser concordantes com os disponíveis na literatura que revelam o predomínio de AF de baixa intensidade $(25,40)$. Quando analisados os valores recolhidos, por sujeito, encontramos uma variabilidade que vai de encontro à investigação que demonstra claramente que, por um lado, há crianças extremamente activas e, por outro, crianças que participam em muito pouca AF $(3,40,41,52)$, verificando-se a existência de subgrupos de crianças que são substancialmente mais activos do que outros.

De facto, os resultados obtidos com esta amostra permitiram verificar que crianças com as mesmas oportunidades de prática de AF evidenciam espontaneamente comportamentos motores diferenciados em quantidade e intensidade, o que reflecte a ideia expressa por diversos investigadores de que há crianças mais predispostas e disponíveis para a prática de AF do que outras (e.g. 65).

Quadro 3: Tempo (minutos) e proporção (\%) de AF de intensidade baixa, moderada e vigorosa, por sexo (média e desviopadrão), valor da estatística t e valor de $p$, no dia escolar.

\begin{tabular}{|c|c|c|c|c|c|c|}
\hline \multirow[b]{2}{*}{ Intensidade } & \multicolumn{2}{|c|}{ Raparigas $[n=14]$} & \multicolumn{2}{|c|}{ Rapazes ( $n=19$ ) } & \multirow[b]{2}{*}{$\mathrm{t}$} & \multirow[b]{2}{*}{$\mathrm{p}$} \\
\hline & Tempo (min) $M \pm D P$ & Prop. [\%] $M \pm D P$ & Tempo $(\mathrm{min}) \mathrm{M} \pm \mathrm{DP}$ & Prop. [\%] $M \pm D P$ & & \\
\hline Baixa & $284.4 \pm 7.1$ & $94.5 \pm 2.4$ & $274.4 \pm 10.2$ & $91.1 \pm 3.4$ & 3.154 & 0.004 \\
\hline Moderada & $14.9 \pm 6.4$ & $5.0 \pm 2.1$ & $23.7 \pm 8.4$ & $7.9 \pm 2.8$ & -3.260 & 0.003 \\
\hline Vigorosa & $1.7 \pm 1.6$ & $0.6 \pm 0.5$ & $2.9 \pm 2.7$ & $1.0 \pm 0.9$ & -1.549 & 0.132 \\
\hline Mod. + Vig. & $16.6 \pm 7.1$ & $5.6 \pm 2.4$ & $26.6 \pm 10.2$ & $8.9 \pm 3.4$ & -3.158 & 0.004 \\
\hline
\end{tabular}


Quando analisados os resultados, por sexo (quadro 3 ), verificam-se diferenças significativas entre ambos os sexos, em dois dos três níveis de intensidade de actividade: as raparigas despendem significativamente mais tempo em AF de baixa intensidade e os rapazes, por seu lado, apresentam valores de $\mathrm{AF}$ moderada e, consequentemente, de AFMV significativamente mais elevados do que as raparigas.

\subsubsection{Resultados obtidos sobre o intervalo escolar} É possível verificar-se no quadro 4 o tempo (em minutos) e a sua proporção (em percentagem), face ao tempo total em que foi considerado que decorria o intervalo, que os alunos despenderam em actividades de intensidade baixa, moderada e vigorosa.

\begin{tabular}{|c|c|c|c|c|}
\hline \multirow[b]{2}{*}{ Variáveis } & \multicolumn{2}{|c|}{ Tempo (minutos) } & \multicolumn{2}{|c|}{ Proporção [\%] } \\
\hline & Média & Desvio-padrão & Média & Desvio-padrão \\
\hline AF Leve & 36.4 & 11.3 & 68.1 & 18.1 \\
\hline AF Moderada & 15.1 & 7.7 & 29.3 & 16.2 \\
\hline AF Vigorosa & 1.3 & 1.5 & 2.5 & 3.0 \\
\hline AFMV & 16.3 & 8.7 & 31.9 & 18.1 \\
\hline
\end{tabular}

A leitura dos seus valores médios percentuais, permite constatar que os sujeitos da amostra despenderam mais tempo, mesmo em período de recreio, em AF de baixa intensidade $(68.1 \pm 18.1 \%)$. O quadro 5 inscreve os resultados obtidos por sexo e o significado da diferença estatística entre os valores obtidos.

Quadro 5: Tempo (minutos) e proporção [\%] de AF leve, moderada e vigorosa, por sexo (média e desvio-padrão), valor da estatística t e valor de $p$, durante o recreio escolar.

\begin{tabular}{lcccc}
\multicolumn{7}{c}{ Raparigas $(\mathrm{n}=14)$} & Rapazes $(\mathrm{n}=19)$ & & \\
Variáveis & Média $\pm \mathrm{DP}$ & Média $\pm \mathrm{DP}$ & $\mathrm{t}$ & $\mathrm{p}$ \\
\hline AF Leve (min) & $40.3 \pm 8.1$ & $33.6 \pm 12.7$ & 1.729 & 0.94 \\
AF Mod. (min) & $11.1 \pm 4.9$ & $18.0 \pm 8.2$ & -2.809 & $\mathbf{0 . 0 0 9}$ \\
AF Vig. (min) & $0.7 \pm 0.8$ & $1.7 \pm 1.7$ & -1.981 & 0.056 \\
AFMV (min) & $11.8 \pm 5.3$ & $19.7 \pm 9.3$ & -2.857 & $\mathbf{0 . 0 0 8}$ \\
\hline AF Leve (\%) & $77.2 \pm 10.8$ & $61.4 \pm 19.7$ & 2.695 & $\mathbf{0 . 0 1 1}$ \\
AF Mod. (\%) & $21.4 \pm 9.8$ & $35.2 \pm 17.6$ & -2.632 & $\mathbf{0 . 0 1 3}$ \\
AF Vig. (\%) & $1.4 \pm 1.6$ & $3.4 \pm 3.5$ & -1.931 & 0.63 \\
AFMV [\%) & $22.8 \pm 10.8$ & $38.6 \pm 19.7$ & -2.695 & $\mathbf{0 . 0 1 1}$
\end{tabular}

A análise dos valores médios permite, assim, confirmar que os sujeitos do sexo masculino apresentam valores significativamente mais elevados de AFMV (38.6 vs. $22.8 \%$ ), quando comparados com os do sexo feminino, nas actividades espontâneas vivenciadas no contexto informal do recreio (55).

3.2.4. Resultados obtidos sobre as aulas de Educação Física A Expressão e Educação Físico-Motora (EF) apresentou uma frequência, em ambas as escolas, de uma aula por semana, com uma duração média de 50 minutos (de 41 a 66 minutos), possibilitando, portanto, o registo de valores de uma aula por cada elemento da amostra. Desde já, é possível referir que esta duração média semanal é muito reduzida, face ao recomendado na literatura internacional $(50,62)$ e também pelo próprio Ministério da Educação que preconiza a realização de duas a três sessões semanais, de 30 a 45 minutos cada, neste ciclo de ensino (32). No quadro 6 temos o tempo (em minutos) e a proporção (em percentagem) da duração total de cada aula que os alunos despenderam em actividades de intensidade baixa, moderada e elevada.

Quadro 6: Tempo (minutos] e proporção [\%] de AF de intensidade baixa, moderada e vigorosa, na totalidade da amostra (média e desvio-padrão) durante as aulas de $\mathcal{E}$.

\begin{tabular}{lcccc} 
& \multicolumn{2}{c}{ Tempo (minutos) } & \multicolumn{2}{c}{ Proporção [\%] } \\
Variáveis & Média & Desvio-padrão & Média & Desvio-padrão \\
\hline AF Leve & 32.7 & 10.3 & 65.9 & 17.3 \\
AF Moderada & 12.7 & 6.2 & 26.5 & 14.1 \\
AF Vigorosa & 4.1 & 5.1 & 7.7 & 9.2 \\
AFMV & 16.8 & 8.2 & 34.1 & 17.3
\end{tabular}

Constata-se que os sujeitos desta amostra despenderam, em média, $66 \%$ do tempo destinado à aula de EF em AF de intensidade baixa e cerca de $34 \%$ em AFMV. No entanto, o quadro 7 permite verificar de que forma estes resultados se apresentaram por sexo e qual o significado da sua diferença estatística. 
Quadro 7: Tempo (minutos] e proporção [\%] de AF leve, moderada e vigorosa, por sexo (média e desvio-padrão), valor da estatística t e valor de $p$, durante as aulas de $\varepsilon F$.

\begin{tabular}{|c|c|c|c|c|}
\hline \multirow[b]{2}{*}{ Variáveis } & Raparigas ( $n=14$ ) & Rapazes ( $n=19$ ) & \multirow[b]{2}{*}{$t$} & \multirow[b]{2}{*}{$\mathrm{p}$} \\
\hline & Média $\pm \mathrm{DP}$ & Média $\pm \mathrm{DP}$ & & \\
\hline AF Leve (min) & $34.6 \pm 10.2$ & $31.4 \pm 10.4$ & 0.882 & 0.385 \\
\hline AF Mod. (min) & 12.1 & $13.1 \pm 7.0$ & -0.411 & 0.684 \\
\hline AF Vig. (min) & 4.6 & \pm 4.7 & 0.529 & 0.601 \\
\hline $\operatorname{AFMV}(\min )$ & 16.8 & $16.7 \pm 9.2$ & 0.017 & 0.987 \\
\hline AF Leve [\%] & 14.2 & $65.1 \pm 19.5$ & 0.312 & 0.757 \\
\hline AF Mod. $[\%]$ & $24.8 \pm 11.7$ & $27.7 \pm 15.9$ & -0.582 & 0.565 \\
\hline AF Vig. $(\%]$ & $8.2 \pm 9.7$ & $7.2 \pm 9.0$ & 0.305 & 0.762 \\
\hline AFMV $[\%]$ & $33.0 \pm 14.2$ & $34.9 \pm 19.5$ & -0.312 & 0.75 \\
\hline
\end{tabular}

O facto de não se verificarem diferenças significativas em função do sexo apoia os resultados de outros estudos em que se verifica que os níveis de AF das raparigas, mesmo de intensidade elevada, se aproximam dos níveis evidenciados pelos rapazes, quando em contexto de aulas estruturadas $(28,59)$. A comparação dos valores obtidos por sexo permite, assim, salientar o importante papel que a EF pode desempenhar na redução das diferenças no envolvimento na $\mathrm{AF}$, defendido por diversos investigadores (e. g. 29), proporcionando às meninas idênticas oportunidades para se envolverem em AF na escola e também promovendo o desenvolvimento de conhecimentos e atitudes que as encorajem, tal como aos rapazes, a fazer da AF regular parte do seu estilo de vida. De facto, os resultados obtidos neste estudo inscrevem, no geral, valores semelhantes aos encontrados em diversos estudos $(28,29,30,51,58,64)$. $\mathrm{Na}$ figura 3 é possível verificar como esta variação se desenhou, por sujeito, em todas as aulas monitorizadas, permitindo salientar que, dos 33 elementos da amostra, apenas $6(18 \%)$ despenderam mais de $50 \%$ da aula de EF em AFMV.

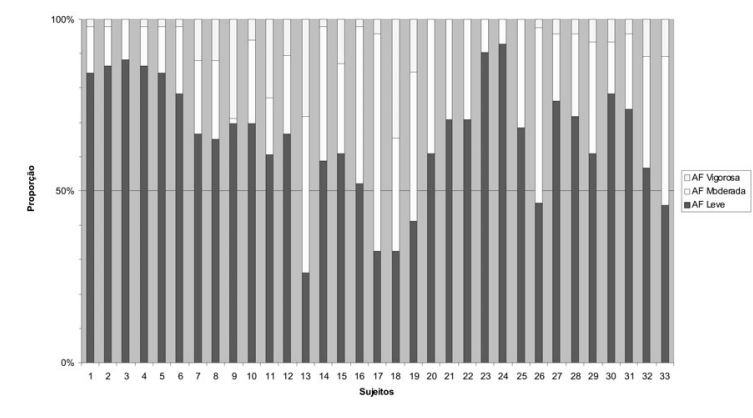
Figura 3: Representação gráfica da variação interindividual da percentagem de tempo despendida em actividades de diferentes intensidades, na aula de $\mathcal{E}$, por sujeito.

Mantém-se, no entanto, a questão da avaliação dos valores obtidos serem ou não suficientes para exercer qualquer tipo de impacto na saúde das crianças a curto ou a longo prazo, dado não haver consenso sobre a quantidade de AF que a criança deve realizar na aula de EF (50). No entanto, estes valores podem ser interpretados à luz de um critério reconhecido que sugere que as crianças se devem manter fisicamente activas, pelo menos, durante $50 \%$ do tempo da aula de EF, o que não terá ocorrido, em média, como já constatado no presente estudo. Assim, poder-se-á induzir que é desejável aumentar o tempo em que as crianças estão envolvidas em AFMV durante as aulas de EF $(34,50)$, o que parece ser possível com simples alterações na gestão destas aulas.

A EF, apesar de apresentar outras finalidades que não, apenas, levar as crianças a participar em AFMV, não pode descurar o seu potencial valor no âmbito dos conteúdos relacionados com a educação para a saúde (57). Por outro lado, a razão, provavelmente mais convincente para que a promoção de estilos de vida activos seja da responsabilidade da escola, nomeadamente no ensino básico, talvez seja o facto de esta ser a única instituição em que praticamente todas as crianças, independentemente da sua condição, têm oportunidade de beneficiar de experiências conducentes a esses mesmos estilos. A promoção de estilos de vida activos é importante também na medida em que exerce uma influência ao nível da prevenção de factores de risco de doenças, facto que não deve ser descurado. 


\subsubsection{Resultados relativos à relação recreio/ aula de Educação Física}

A comparação entre os resultados obtidos no tempo de recreio e na aula de EF pode ser verificada no quadro 8 .

Quadro 8: Tempo (minutos] e proporção [\%] de AF leve, moderada e vigorosa, por sexo (média e desvio-padrão), valor da estatística t e valor de $p$, durante o recreio e as aulas de $\varepsilon F$.

\begin{tabular}{|c|c|c|c|c|}
\hline & Recreio & Educação Física & & \\
\hline Variáveis & Média $\pm \mathrm{DP}$ & Média $\pm \mathrm{DP}$ & $\mathrm{t}$ & $\mathrm{p}$ \\
\hline AF Leve (min) & $36.4 \pm 11.3$ & $32.7 \pm 10.3$ & -1.388 & 0.17 \\
\hline AF Mod. (min) & 15.1 & 12.7 & -1.391 & 0.169 \\
\hline AF Vig. (min) & $1.3 \pm 1.5$ & $4.1 \pm 5.1$ & 3.065 & 0.00 \\
\hline AFMV $(\min )$ & $16.3 \pm 8.7$ & $16.8 \pm 8.2$ & 0.207 & 0.83 \\
\hline AF Leve $[\%]$ & $68.1 \pm 18.1$ & $65.9 \pm 17.3$ & -0.514 & 0.60 \\
\hline AF Mod. $(\%)$ & $29.3 \pm 16.2$ & $26.5 \pm 14.1$ & -0.768 & 0.44 \\
\hline AF Vig. $(\%]$ & $2.5 \pm 3.0$ & $7.7 \pm 9.2$ & 3.038 & 0.003 \\
\hline AFMV $[\%]$ & $31.9 \pm 18.1$ & $34.1 \pm 17.3$ & 0.514 & 0.60 \\
\hline
\end{tabular}

Constata-se que o comportamento dos alunos em situação livre de recreio ou organizada na aula de EF apenas revelou ser significativamente diferente quanto à realização de $\mathrm{AF}$ intensa, onde se registaram valores médios superiores durante as aulas de EF (4.1 vs. $1.3 \%$ ) em relação ao tempo de recreio. No entanto, a AFMV, no seu conjunto, foi semelhante nos dois contextos (16.3 e $16.8 \%$ ).

Estes resultados são díspares dos verificados por Sleap e Warburton (58) que constataram que os maiores registos de AFMV ocorrem durante o recreio (em cerca de $50 \%$ do tempo total observado), quando comparados com os obtidos nas aulas de EF (cerca de $40 \%$ ).

Persiste a dúvida sobre se é pertinente a preocupação de diversos investigadores com a criança que apresenta baixos níveis de AF. No entanto, confirma-se a ideia de que há crianças espontaneamente muito activas e outras que realizam voluntariamente muito pouca AF. Se considerarmos, igualmente, que há estudos que demonstram que a criança apresenta níveis inferiores de AF na ocupação dos seus tempos livres fora da escola (58) e que a infância é considerada como o estádio da vida mais activo, a partir do qual se assiste a um decréscimo nos níveis de AF, parece-nos pertinente a realização de mais investigação neste âmbito, que permita uma intervenção efec- tiva junto das crianças. Mesmo considerando que a participação diária em AF seja baixa, o envolvimento escolar, através das aulas de EF e a oportunidade para jogar e brincar no recreio, pode proporcionar mais oportunidades para que as crianças sejam activas. Consideramos, no entanto, que é necessário averiguar muitos outros aspectos das aulas de EF que não se circunscrevem ao aumento dos níveis de AF das crianças. Este estudo não pretendeu avaliar os conteúdos e matérias leccionadas nas aulas monitorizadas, onde, segundo apurámos, predominaram os jogos infantis, nem as mensagens relacionadas com a saúde transmitidas pelos seus professores; mesmo notando que as crianças, de uma maneira geral, gostavam e ansiavam por estas aulas, não avaliámos estas questões. No entanto, sabemos que estas são questões igualmente importante na $\mathrm{AF}$ relacionada com a saúde.

\section{CONCLUSÕES}

Da análise e discussão dos resultados emerge o seguinte quadro de conclusões:

As crianças do sexo feminino evidenciam menores níveis de AF, essencialmente de intensidade elevada, na ocupação dos seus tempos livres. De facto, encontramos nos rapazes resultados significativamente superiores no valor compósito de actividade física no tempo de lazer e no relativo à actividade extenuante, quando comparados com os das raparigas.

As crianças apresentam um padrão espontâneo de actividade física complexo e de natureza transitória, distinto do adulto. A representação gráfica do dispêndio energético ocorrido durante os períodos de recreio permitiu-nos confirmar o carácter aleatório e irregular com que se evidenciam comportamentos de diferentes intensidades, não persistindo durante extensos períodos de tempo a realização de actividades de intensidade elevada.

A avaliação de actividade física realizada pelas crianças, durante o tempo escolar, através do Tritrac-R3D, permitiu confirmar a ideia empírica de que as crianças pertencentes à nossa amostra despendem grande proporção do tempo passado na escola sentadas na sala de aula, onde evidenciam níveis de actividade física sedentária ou com ligeiras oscilações de actividade física de muito baixa intensidade, verificandose um predomínio da actividade física leve. Esta correspondeu a $92,6 \%$ do tempo total despendido na escola, enquanto a actividade física moderada a vigorosa ocorreu, em média, em apenas $7.4 \%$ desse 
tempo. Esta percentagem corresponde a cerca de 22 minutos, não atingindo, no mínimo, a meia hora de actividade física moderada a vigorosa recomendada por diversas directrizes internacionais.

Quando analisados os resultados relativos à realização espontânea de actividade física no contexto informal do recreio, verificou-se que os elementos do sexo feminino despenderam uma proporção do seu tempo significativamente superior em actividade física leve, quando comparados com os do sexo masculino. Estes, por seu lado, apresentam valores médios significativamente superiores no respeitante à actividade física moderada e, consequentemente, à actividade física moderada a vigorosa.

A análise dos resultados obtidos, durante as aulas de Educação Física, revelou, em termos da proporção de tempo despendido em actividade física de diferentes intensidades, valores próximos dos obtidos por vários estudos internacionais, em que, mesmo utilizando metodologias distintas, a maior percentagem de tempo da aula se verificou ser despendida em actividade física sedentária ou leve. No entanto, a percentagem média de tempo despendida em actividade física moderada a vigorosa por esta amostra (34.1\%) não atingiu o critério recomendado por uma instituição de renome internacional americana que preconiza que ao alunos devem despender, pelo menos, metade do tempo da aula de Educação Física em actividade física significativa (62).

Quando comparados os resultados obtidos pelas crianças durante as aulas de Educação Física com aqueles obtidos no recreio, verifica-se que apenas se revelaram significativamente diferentes quanto à realização de actividade física intensa. No entanto, quando analisada no seu conjunto, a actividade física moderada a vigorosa, assim como, a actividade física de baixa intensidade registaram valores próximos nos dois contextos, não se podendo, portanto, inferir que, no geral, se tenha verificado um comportamento diferente quanto ao dispêndio energético.

\section{CORRESPONDÊNCIA}

José Maia

Laboratório de Cineantropometria

e Estatística Aplicada

Faculdade de Ciências do Desporto

e de Educação Física

Universidade do Porto

Rua Dr. Plácido Costa, 91

4200.450 Porto

Portugal

jmaia@fcdef.up.pt 


\section{BIBLIOGRAFIA}

1. Armstrong N, Balding J, Bray S, Gentle P, Kirby B (1990). The physical activity patterns of 10 and 13 year old children. In: G Beunen, J Ghesquire, T Reybrouk, A Classens (eds.). Children and exercise. Stuttgart: Enke, 152-157.

2. Armstrong N, Bray S (1991). Physical activity patterns defined by continuous heart rate monitoring. Archives of Disease in Childhood 66: 245-247.

3. Armstrong N, Van Mechelen W (1998). Are young people fit and active? In: S Biddle, J Sallis, N Cavill (eds.). Young and active? Young people and health-enhancing physical activity evidence and implications. London: Health Education Authority, 69-97.

4. Armstrong N, Williams J, Balding J, Gentle P, Kirby B (1991). Cardiopulmonary fitness, physical activity patterns, and selected coronary risk factor variables in 11- to 16- year olds. Pediatric Exercise Science 3: 219-228.

5. Bailey R, Olson J, Pepper S, Porszasz J, Barstow T, Cooper D (1995). The level and tempo of children's physical activities: an observational study. Medicine $\mathcal{E}$ Science in Sports $\mathcal{E}$ Exercise 7: 1033-1041

6. Barbosa T, Magalhães P, Lopes V (1999). Gender, socioeconomic status and maturation differences in pubertal children's physical activity. Poster apresentado no Pediatric Work Physiology, Roma.

7. Biddle S, Goudas M (1996). Analysis of children's physical activity and its association with adult encouragement and social cognitive variables. Journal of School Health 66(2): 75-78.

8. Biddle S, Sallis J Cavill N (1998). Policy framework for young people and health-enhancing physical activity. In: $\mathrm{S}$ Biddle, J Sallis, N Cavill (Eds.). Young and active? Young people and health-enhancing physical activity - evidence and implications. London: Health Education Authority, 3-16.

9. Blair S (1992). Are American children and youth fit? The need for better data. Research Quarterly for Exercise and Sport 63(2): 120-123.

10. Blair S (1993). C. H. McCloy research lecture: physical activity, physical fitness, and health. Research Quarterly for Exercise and Sport 64 (4): 365-376.

11. Bradley C, McMurray R, Harrell J, Deng S (2000). Changes in common activities of 3rd through 10th graders: the CHIC study. Medicine \& Science in Sports \& Exercise 32(12) 2071-2078.

12. Cale L, Almond L (1997). The physical activity levels of English adolescent boys. European Journal of Physical Education 2: 74-82.

13. Caspersen C, Nixon P, DuRant R (1998). Physical activity epidemiology applied to children and adolescents. Exercise and Sport Sciences Reviews 26: 341-403.

14. Cavill N, Biddle S, Sallis J (2001). Health enhancing physical activity for young people: statement of the United Kingdom expert consensus conference. Pediatric Exercise Science 13: 12-25.

15. Centers for Disease Control and Prevention (CDC) (1997). Guidelines for school and community programs to promote lifelong physical activity among young people. Morbidity \& Mortality Weekly Report. National Center for Chronic Disease Prevention and Health Promotion. Online at http://www.cdc.gov/nccdphp/dash/phactaag.htm

16. Coetzee N (2000). Man and his health. International Journal of Physical Education 37(3): 103-111.
17. Corbin C, Pangrazi R (1996). How much physical activity is enough? JOPERD 67(4): 33-37.

18. Freedman D, Dietz W, Srinivasan S, Berenson G (1999). The relation of overweight to cardiovascular risk factors among children and adolescents: The Bogalusa Heart Study. Pediatrics 103(6): 1175-1182.

19. Gilbey H, Gilbey M (1995). The physical activity of Singapore primary school children as estimated by heart rate monitoring. Pediatric Exercise Science 7: 26-35.

20. Gilliam T, MacConnie S (1984). Coronary heart disease risk in children and their physical activity patterns. In: $\mathrm{R}$ Boileau (Ed.). Advances in Pediatric Sport Sciences, Volume 1, Champaign, IL: Human Kinetics, 171-187.

21. Godin G, Shepard R (1985). A simple method to assess exercise behavior in the community. Can. J. Appl. Sport Science 10 (3): 141-146.

22. Gutin B, Owens S (1996). Is there a scientific rationale supporting the valeu of exercise for the present and future cardiovascular health of children? The pro argument. Pediatric Exercise Science 8: 294-302.

23. Hovell M, Sallis J, Kolody B, McKenzie T (1999). Children's physical activity choices: a developmental analysis of gender, intensity levels, and time. Pediatric Exercise Science 11: 158-168.

24. Janz K, Witt J, Mahoney L (1995). The stability of children's physical activity as measured by accelerometry and self-report. Medicine \& Science in Sports \& Exercise 27(9): 1326-1332.

25. Kelly L (2000). Patterns of physical activity in $9-10-$ year - old American children as measured by heart rate monitoring. Pediatric Exercise Science 12: 101-110.

26. Maia J (Ed.) (2001). Genética e práticas desportivo-motoras Actas do Seminário. Porto.

27. Maia J, Lopes V (2001). Actividade física e aptidão física associada à saúde. Um estudo de epidemiologia genética em gémeos e suas famílias, realizado no Arquipélago dos Açores. DREFD e FCDEF.

28. McKenzie T, Feldman H, Woods S, Romero K, Dahlstrom V, Stone E, Strikmiller P, Williston J, Harsha D (1995). Children's activity levels and lesson context during thirdgrade Physical Education. Research Quarterly for Exercise and Sport 66(3): 184-193.

29. McKenzie T, Nader P, Strikmiller P, Yang M, Stone E, Perry C, Taylor W, Epping J, Feldman H, Luepker R, Kelder S (1996). School Physical Education: effect of the Child and Adolescent Trial for Cardiovascular Health. Preventive Medicine 25: 423-431.

30. McKenzie T, Sallis J, Faucette N, Roby J, Kolody B (1993). Effects of a curriculum and inservice program on the quantity and quality of elementary physical education classes. Research Quarterly for Exercise and Sport 64(2): 178-187.

31. Miller D, Freedson P, Kline G (1994). Comparison of activity levels using the Caltrac accelerometer and five questionnaires. Medicine \& Science in Sports \& Exercise 26(3): 376-382.

32. Ministério da Educação (1992). A Educação Física no $10^{\circ}$ Ciclo. Lisboa: Ministério da Educação.

33. Montoye H, Kemper H, Saris W, Washburn R (1996). Measuring physical activity and energy expenditure. Champaign: Human Kinetics Publishers.

34. Morris H (1991). The role of school physical education in public health. Research Quarterly for Exercise and Sport 62(2): 143-147. 
35. Myers L, Strikmiller P, Webber L, Berenson G (1996). Physical and sedentary activity in school children grades 58: the Bogalusa Heart Study. Medicine $\mathcal{E}$ Science in Sports $\mathcal{E}$ Exercise 28(7): 852-859.

36. Oja P (1995). Descriptive epidemiology of health-related physical activity and fitness. Research Quarterly for Exercise and Sport 66(4): 303-312.

37. Pangrazi R, Corbin C, Welk G (1996). Physical activity for children and youth. JOPERD 67(4): 38-43.

38. Pate R (1995). Physical activity and health: dose-response issues. Research Quarterly for Exercise and Sport 66(4): 313-317.

39. Riddoch C (1998). Relationships between physical activity and health in young people. In: S Biddle, J Sallis, N Cavill (Eds.). Young and active? Young people and health-enhancing physical activity - evidence and implications. London: Health Education Authority, 17-48.

40. Riddoch C, Boreham C (1995). The health-related physical activity of children. Sports Medicine 19(2): 86-102.

41. Riddoch C, Mahoney C, Murphy N, Boreham C, Cran G (1991). The physical activity patterns of Northern Irish schoolchildren ages 11-16 years. Pediatric Exercise Science 3: 300-309.

42. Rowland T (1990). Exercise and children's health. Champaign, IL: Human Kinetics.

43. Saakslahti A, Numminen P, Niinikoski H, Rask-Nissila L, Viikari J, Tuominen J, Valimaki I (1999). Is physical activity related to body size, fundamental motor skills, and $\mathrm{CHD}$ risk factors in early childhood? Pediatric Exercise Science 11: 327-340.

44. Sallis J (1995). A behavioral perspective on children's physical activity. In: L Cheung, J Richmond (Eds.). Child health, nutrition, and physical activity. Champaign IL: Human Kinetics, 125-138.

45. Sallis J (1987). A commentary on children and fitness: a Public Health perspective. Research Quarterly for Exercise and Sport 58(4): 326-330.

46. Sallis J (1995). A North American perspective on physical activity research in children and adolescents. In C Blimkie, O Bar-Or (Eds.). New Horizons in Pediatric Exercise Science. Champaign IL: Human Kinetics, 221-234.

47. Sallis J (1994). Determinants of physical activity behavior in children. In: R Pate \& R Hohn (eds.). Health and fitness through Physical Education. Champaign IL: Human Kinetics, 31-43.

48. Sallis J, Alcaraz J, McKenzie T, Hovell M, Kolody B, Nader $P$ (1992). Parental behavior in relation to physical activity and fitness in 9-year-old children. American Journal Diseases of Children 146: 1383-1388.

49. Sallis J, Buono M, Roby J, Micale F, Nelson J (1993). Seven-day recall and other physical activity self-reports in children and adolescents. Medicine $\mathcal{E}$ Science in Sports $\mathcal{E}$ Exercise 25(1): 99-108.

50. Sallis J, McKenzie T (1991). Physical Education's role in Public Health. Research Quarterly for Exercise and Sport 62 (2): 124-137.

51. Sallis J, McKenzie T, Alcaraz J, Kolody B, Faucette N, Hovell M (1997). The effects of a 2-year Physical Education Program (SPARK) on physical activity and fitness in elementary school students. American Journal of Public Health 87(8): 1328-1334.
52. Sallis J, McKenzie T, Elder J, Hoy P, Galati T, Berry C, Zive $M$, Nader P (1998). Sex and ethnic differences in children's physical activity: discrepancies between self-report and objectives measures. Pediatric Exercise Science 10: 277-284.

53. Sallis J, Prochaska J, Taylor W (2000). A review of correlates of physical activity of children and adolescents. Medicine \& Science in Sports \& Exercise 32(5): 963-975.

54. Saris W (1986). Habitual physical activity in children: methodology and findings in health and disease. Medicine \& Science in Sports \& Exercise 18(3): 253-263.

55. Sarkin J, Nichols J, Sallis J, Calfas K (2000). Self-report measures and scoring protocols affect prevalence estimates of meeting physical activity guidelines. Medicine $\mathcal{E}$ Science in Sports \& Exercise 32(1): 149-156.

56. Shephard R, Trudeau F (2000). The legacy of Physical Education: influences on adult lifestyle. Pediatric Exercise Science 12: 34-50.

57. Sleap M (1990). Promoting health in primary school physical education. In N Armstrong (ed.). New Directions in Physical Education, Volume 1. Champaign, IL: Human Kinetics, 17-34.

58. Sleap M, Warburton P (1996). Physical activity levels of 511-year-old children in England: cumulative evidence from three direct observation studies. International Journal of Sports Medicine 17(4): 248-253.

59. Strand B, Walswick P, Sommer C (2000). Tracking children's caloric expenditure in Physical Education. JOPERD 71(5): 35-39.

60. Thomas J, Thomas K (1988). Development of gender differences in physical activity. Quest 40: 219-229.

61. Twisk J (2000). Physical activity, physical fitness and cardiovascular health. In: N Armstrong e $\mathrm{W}$ van Mechelen (eds.). Paediatric exercise science and medicine. Champaign IL: Human Kinetics, 253-264.

62. U.S. Department of Health and Human Services (1991). Healthy People 2000: national health promotion and disease prevention objectives. Washington DC: Department of Health and Human Services, Government Printing Office.

63. U.S. Department of Health and Human Services (2000). Healthy People 2010: understanding and improving health. Washington DC: Department of Health and Human Services, Government Printing Office. Online at http://www.health.gov/healthy

64. Warburton P, Woods J (1996). Observation of children's physical activity levels during primary school physical education lessons. European Journal of Physical Education 1: 56-65.

65. Wood K, Morss G, Fernandez J, Gill D, Welk G (2000). Variability and stability of physical activity patterns in children. Research Quarterly for Exercise and Sport 71 (Suppl.): A35. 